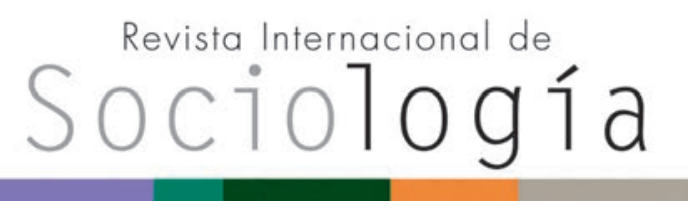

Revista Internacional de Sociología RIS vol. 75 (4), e074, octubre-diciembre, 2017, ISSN-L:0034-9712 doi: http://dx.doi.org/10.3989/ris.2017.75.4.17.07

\section{SOCIOLOGÍAS DE LA ENERGÍA Hacia una agenda de investigación}

\author{
TOMÁs ARIZTíA
}

Universidad Diego Portales, Chile.

tomas.ariztia@udp.cl

ORCID iD: http://orcid.org/0000-0001-5806-3328

ÀLEX Boso

Universidad de la Frontera, Chile.

alex.boso@ufrontera.cl

ORCID iD: http://orcid.org/0000-0002-0313-8238

MANUEL TIRONI

Pontificia Universidad Católica de Chile, Chile.

metironi@uc.cl

ORCID iD: http://orcid.org/0000-0003-2733-8071

Cómo citar este artículo / Citation: Ariztía, T., A. Boso, y M. Tiron. 2017. "Sociologías de la energía. Hacia una agenda de investigación". Revista Internacional de Sociología 75 (4): e074. doi: http://dx.doi.org/10.3989/ ris.2017.75.4.17.07

\section{RESUMEN}

El presente artículo presenta una visión panorámica de los estudios sociales de la energía a la vez que introduce los artículos del monográfico. Se comienza presentando las razones que explican la progresiva visibilidad de las temáticas energéticas en la sociología, aludiendo al desafío que impone el cambio climático y la creciente atención de las ciencias sociales hacia la dimensión material de la vida social. Se propone entender la energía como un objeto de estudio de carácter esencialmente socio técnico, esto es, como un fenómeno que exige una aproximación que dé cuenta de los aspectos materiales, técnicos, sociales y culturales que lo constituyen. El artículo presenta luego diferentes áreas de investigación relevantes para el debate sociológico sobre la intersección entre energía y sociedad y su relación con las contribuciones al monográfico. Los estudios que se presentan no sólo tienen interés para la comunidad académica del área de la sociología, sino que pueden ser de utilidad para profesionales de la energía e investigadores de otras disciplinas interesados en abordar los aspectos sociales de la energía.
\end{abstract}

\section{Palabras Clave}

Aceptación social; Energía y sociedad; Estudios energéticos; Implicación; Prácticas sociales; Tecnologías energéticas emergentes.

\section{SOCIOLOGIES OF ENERGY} Towards a research agenda
Copyright: @ 2017 CSIC. Este es un artículo de acceso abierto distribuido bajo los términos de la licencia Creative Commons Attribution (CC BY) España 3.0.

Recibido: 15/12/2016. Aceptado: 10/07/2017

\section{Abstract}

This article offers a panoramic view of the field of the social studies of energy while introducing the articles of the special issue. It begins by discussing the progressive interest on studying the social aspects of energy. We relate this interest to the increasing challenges imposed by global climate change as well as the growing sociological attention to the material dimension of social life. The article suggests understanding energy and energy related phenomena as a socio-technical object which involve material, social, cultural and technical elements. The article then briefly describes different research areas concerning the intersection between energy and society and present the contributions to the monograph. We suggest that the articles comprised in this special issue are not only relevant for social scientist interested on energy related issues; they might also help energy professionals and researchers from outside the social sciences to further problematize the social aspects and challenges of energy.

\section{KeYwords}

Emerging energy technologies; Energy and society; Energy studies research; Engagement; Social acceptance; Social practices. 


\section{ENERGIZANDO LA SOCIOLOGÍA}

En la primera página de su famoso tratado Las reglas del método sociológico, Emile Durkheim (2001[1895]) observaba que en las sociedades contemporáneas "cada individuo bebe, duerme, come, razona y a la sociedad le interesa que dichas funciones se ejerzan en forma regular" (Íbid., 38). Estas funciones soportan la vida social, pero en tanto que parte de la mecánica técnica, biológica o fisicoquímica de la existencia, se apura en aclarar el sociólogo francés, no son ni pueden ser el objeto de la sociología. A este hay que ir a buscarlo a otra parte. "En todas las sociedades", indica Durkheim, "existe un grupo determinado de fenómenos que se distinguen marcadamente de los que estudian las ciencias de la naturaleza" (íbid.), las encargadas de investigar los procesos materiales que no tienen ni origen ni intención social.

Durante décadas, esta demarcación fenomenológica caló hondo en el campo epistémico de la sociología. Esta estudiaba la familia, la política o el trabajo, fenómenos formados en, por y para la vida colectiva humana, quedando en manos de la ingeniería o las ciencias naturales el análisis del mundo biológico, vegetal o mineral. Sin embargo, en los últimos años, diversas sociologías comienzan a reconocer el rol de diferentes elementos no "sociales" en la constitución de lo social, y a preguntarse por las especificidades materiales del fenómeno social. Tanto es así que, en el centro de la disciplina, empiezan a ubicarse objetos de investigación que Durkheim jamás hubiese catalogado de sociológicos.

El presente número especial intenta introducir en la sociología de habla hispana uno de ellos: la energía. La Real Academia Española define energía como "Eficacia, poder, virtud para obrar", lo que desde una perspectiva física se traduce a "Capacidad para realizar un trabajo" (RAE 2017, n/p). Por tanto, la energía, entendida como combustible o fuerza eléctrica proveniente de alguna fuente de generación, es un objeto y proceso que opera en las sombras de la vida social, subterráneamente, entregando "capacidad para realizar" lo social -dotando la base material para que la vida normal en occidente, el dormir, comer y beber de Durkheim, siga su cursopero sin ser ella misma social. La energía sería una infraestructura, es decir, parte de los mecanismos invisibles detrás de otros tipos (más importantes) de trabajo (Larkin 2013).

Sin embargo, la imagen de la energía como infraestructura se vuelve más compleja desde la perspectiva de su situación social y de su capacidad para "actuar" en la vida colectiva (cf. Star y Ruhleder 1996; Star 1999; Barry 2001; Edwards 2003; Jensen y Winthereik 2013). Siguiendo esta sensibilidad infraestructural y situacional, las discusiones energéticas han cobrado una relevancia significativa en la in- vestigación social y, especialmente, en la sociología durante los últimos años (Guy y Shove 2000; Zélem y Beslay 2015). Las causas de este renovado foco de atención son variadas, así como las tradiciones teóricas que sostienen este giro. El visible desafío que impone el cambio climático y la necesidad de potenciar una transición hacia una era post-carbón ha incrementado el interés de la sociología, particularmente desde la perspectiva de la innovación responsable y la ciencia y tecnología, en los desafíos sociales de las energías renovables no-convencionales y su rol en el desarrollo sustentable (Stern 2014; Upham et al. 2015; Zélem y Beslay 2015).

A este interés se le suma la proliferación de conflictos ciudadanos en torno a proyectos energéticos, tanto en América Latina (Varas et al. 2013; Tironi y Barandiaran 2014) como en América del Norte y Europa (McAdam y Boudet 2012; Rule 2014; DevineWright 2015). Este posicionamiento como objeto privilegiado de conflictos socioambientales ha ubicado a la energía en el radar de la sociología medioambiental y de los movimientos sociales.

Por último, la creciente centralidad que ocupa la energía en la organización de un repertorio amplio de procesos sociales, tales como la economía, el transporte, la vida doméstica y urbana, en definitiva, en la configuración y estabilización de la "normalidad", han posicionado a la energía como tema de investigación dentro de los estudios sociales de la ciencia y tecnología y de la sociología de las prácticas.

\section{ESTUDIOS SOCIALES DE LA ENERGÍA: UNA MIRADA PANORÁMICA AL CAMPO}

La agenda de investigación en torno a la energía -desarrollada principalmente en el mundo académico de habla inglesa- se puede agrupar en torno a al menos seis grandes ámbitos temáticos.

Un primer foco de atención ha estado en el estudio de grandes infraestructuras y sistemas sociotécnicos y su relación con distintos colectivos y procesos sociales. Principalmente, desde la sociología de la ciencia y tecnología, se ha investigado la red de supuestos, técnicas, conocimientos y traducciones que configuran el diseño y la implementación de infraestructuras energéticas (Summerton 2004). Esta agenda de investigación se ha centrado en estudiar los procesos de desarrollo e instalación de grandes sistemas sociotécnicos, como los sistemas eléctricos, en relación al despliegue de dinámicas de transformación sociopolítica vinculados al desarrollo de formas específicas de expansión del capitalismo y la democracia (Hughes 1983).

Una segunda área de investigación de particular relevancia tiene relación con el estudio de los conflictos asociados al desarrollo de infraestructuras energéticas y su relación con comunidades y colec- 
tivos afectados. La sociología de la acción colectiva y los movimientos sociales, por ejemplo, ha investigado cuáles son las lógicas de los conflictos que los proyectos energéticos suscitan (McAdam y Boudet 2012). En esta misma línea, la sociología se ha mostrado recientemente interesada en problematizar los formatos clásicos de la participación y la representación asociadas a estas infraestructuras (Wilkie et al. 2015), reformulando de paso las comprensiones tradicionales de las relaciones entre saberes expertos y legos (Marres 2012).

En tercer lugar, y volviendo a la discusión sobre la composición no-social de lo social, un importante cuerpo de investigación, inspirado en la teoría del actor-red, el nuevo materialismo y otras filosofías ontológicas, se ha enfocado en estudiar el modo en que las especificidades fisicoquímicas de la energía ayudan a explicar y modelar situaciones sociopolíticas. El trabajo de Mitchell (2011) sobre el rol de las características geológicas, químicas y técnicas del petróleo en la configuración de la democracia occidental o la investigación de Hecht (2009) sobre la articulación entre las características técnicas de la energía nuclear y la formación del estado francés contemporáneo son ejemplos de esta agenda. Otros aportes relevantes en este ámbito de investigación son los trabajos de Barry (2015) y Howe y Boyer (2015) sobre la termodinámica como modo de pensar el nexo energía-política y la implicación de las condiciones eólicas sobre la articulación de la política "sustentable" en México, respectivamente.

Un cuarto foco de interés se relaciona con el abordaje de las prácticas e infraestructuras domésticas asociadas al uso (y, crecientemente, producción) de energía (Shove 2010a; Shove 2010b). Esta perspectiva, que surge principalmente de la intersección entre la sociología del consumo y la sociología de la ciencia y tecnología, se ha centrado en analizar las prácticas y tecnologías a partir de las cuales se organiza el uso de energía en el ámbito doméstico (Ariztía 2017). La mirada sociológica aquí, revela la relación de la energía con la organización social de la vida cotidiana y sus distintas formas de intersección con infraestructuras y entramados sociotécnicos de mayor escala. Utilizando el concepto de "práctica" como eje analítico, estos estudios han contribuido a mostrar las limitaciones que subyacen a enfoques tradicionales de corte más individualista. Diversos estudios muestran que es preciso analizar el uso de energía como resultado de la organización cotidiana de prácticas, rutinas e infraestructuras existentes y los distintos supuestos normativos y funcionalidades (affordances) que estos movilizan (McMeekin y Southerton 2012; Shove y Walker 2014).

Un quinto foco se ha centrado en estudiar I los procesos de economización y valuación asociados a la energía. En este contexto, la energía se ha transformado en un caso de estudio particularmente fértil para estudiar las dinámicas sociológicas que subyacen a la construcción de objetos de regulación, procesos y formas de experimentación económica (Alvial-Palavicino y Ureta 2017). Así emerge un campo de estudio de particular relevancia para la sociología como es la imbricación entre políticas de transición energética y la creación de nuevos mercados, productos y formas económicas (Blok 2011; Pallesen 2016) y su relación con modos específicos de evaluación (MacKenzie 2009).

Finalmente, es posible observar un sexto foco cercano al esfuerzo interdisciplinario por pensar, desarrollar y evaluar críticamente los procesos de transición hacia sociedades post-carbón (Berti y Levidow 2014; Geels 2014; Geels et al. 2016) y en particular procesos de transición energética (Cointe 2015). Buena parte de esta agenda ha estado asociada a una creciente revalorización de la sociología y del saber de las ciencias sociales como un aspecto central en la comprensión del diseño, modificación y gobierno de sistemas energéticos en el marco de los desafíos que impone el cambio climático (Geels 2011; Spaargaren 2011; McMeekin y Southerton 2012).

\section{LAS CONTRIBUCIONES: OBJETOS SOCIOTÉCNICOS Y UNA MIRADA DESDE LAS FORMAS DE IMPLICACIÓN}

Los artículos de este monográfico están cruzados por muchas de las perspectivas presentadas sucintamente en los párrafos anteriores. Asimismo, los artículos que componen este número especial reflejan la creciente porosidad disciplinar, temática y regional que constituye el campo de los estudios sociales de la energía que acabamos de describir. De hecho, representan distintas geografías, contextos socioculturales, objetos de estudio, metodologías y aproximaciones teóricas.

Con todo, una lectura transversal de estas contribuciones permite identificar que en esta diversidad teórica, metodológica y territorial emergen algunas hebras comunes. Creemos que estas pueden constituir anclajes para un programa de investigación sociológico sobre la energía.

Un primer aspecto común está vinculado a la naturaleza de la energía como objeto de estudio. Todos los artículos de este monográfico abordan la energía como un objeto eminentemente sociotécnico. Por sociotécnico se entienden objetos o procesos que requieren tanto de explicaciones sociales como técnicas, sin la imposición de una sobre la otra, para entender su conformación, estabilización o funcionamiento (Callon 1980). Siguiendo esta línea, los artículos que aquí se presentan analizan la energía -la forma en la que la producimos, distribuimos y usamos- como un fenómeno que es a su vez infraestructural y cultural, material y simbólico, físico y 
Figura 1.

La energía como objeto sociológico: seis agendas de investigación.

SISTEMAS SOCIO-

TÉCNICOS

Sociología Ciencia y

Tecnología

innovacion Responsadie
CONFLICTOS

ENERGÉTICOS

Sociología Medioambiental

Sociología Acción Colectiva

Sociología Movimientos Sociales

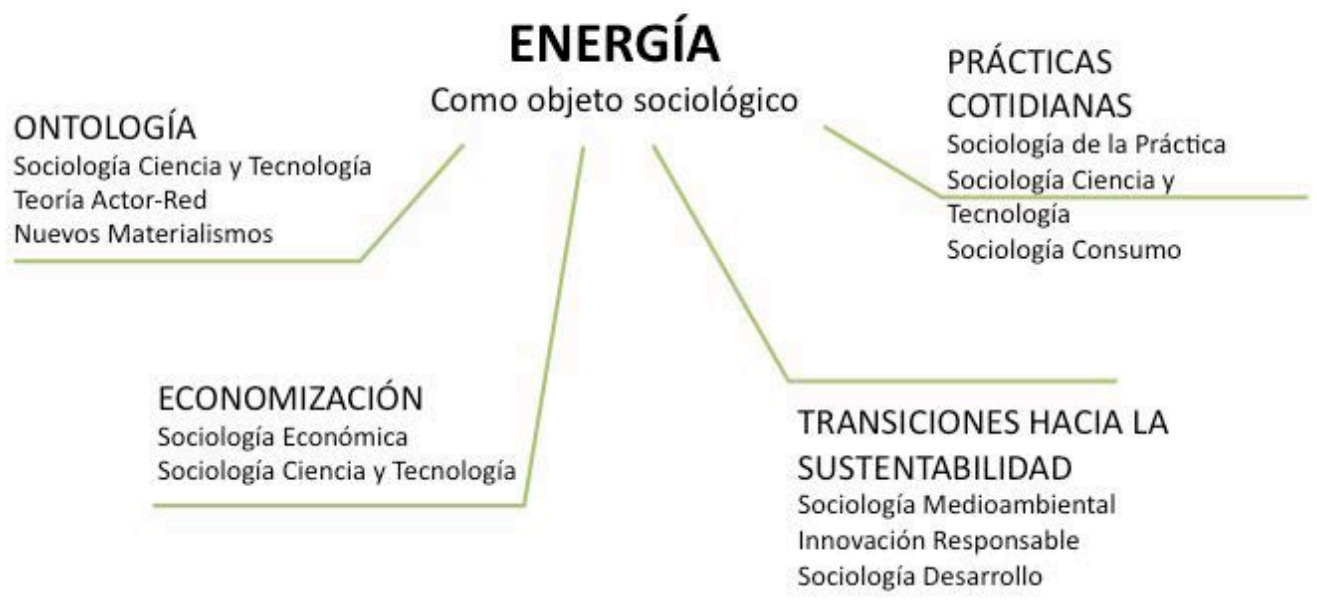

Fuente: Elaboración propia.

político. Si bien esta constatación es ya un punto de partida común en los estudios sociales de la ciencia y tecnología, lo interesante de los trabajos que aquí se presentan es que muestran esta naturaleza sociotécnica en acción de distintas formas, a distintas escalas y formas.

Por ejemplo, Gross y Tironi analizan en su artículo la prospección geotérmica como un tipo particular de experimento que se da simultáneamente en el campo de la geoingeniería y en el ciudadano. Según estos autores, la experimentalidad de la geotermia hace imposible separar los elementos técnicos de los políticos, a la vez que plantea nuevos retos y preguntas sobre la relación entre la producción de ignorancia, transiciones sustentables y democracia. En esta misma línea, Tironi y Sannazzaro se aproximan con una mirada sociológica a un proyecto eólico en la isla de Chiloé, en el sur de Chile. En su análisis tratan a este proyecto eólico como un tipo de infraestructura para la generación de energía renovable. Pero a su vez, sostienen los autores, esta infraestructura opera como un dispositivo político relacionado con la inclusión de los pueblos originales en la economía nacional. En ambos estudios la energía es presentada como un elemento híbrido que genera consecuencias (y ancla sus causas) tanto en ámbitos técnicos como sociales.

La energía nuclear es una tecnología tan compleja como socialmente controvertida. Espluga et al. discuten cómo las características sociotécnicas de esta energía provocan una serie de riesgos perci- bidos tanto en los actores sociales encargados de su gobernanza, como en el público no experto que, a su vez, son motor de controversias sociales. Por otro lado, el artículo de Oltra et al. estudia la aceptación social de una tecnología energética innovadora como son las pilas combustible de hidrógeno para el ámbito doméstico o el transporte. El análisis de las actitudes hacia un elemento sociotécnico (desconocido por gran parte de la población) es clave para plantear diferentes barreras y oportunidades en un escenario de transición energética. De forma similar, el artículo de Boso, Ariztía y Fonseca propone que para el estudio de las políticas de recambio de las estufas a leña, es necesario observar cómo interaccionan distintas prácticas, materialidades e infraestructuras en el ámbito cotidiano, para entender los mecanismos de bloqueo que ponen freno a la implementación eficaz de estas políticas.

Pero, ¿cómo y desde dónde se pueden analizar los distintos objetos sociotécnicos? Los trabajos aquí publicados comparten un segundo elemento en la manera de abordar sus objetos de estudio: observar y estudiar las formas de implicación que propone la energía hacia el mundo social. Por implicación entendemos la particular serie de relaciones creada en o por distintas formas de generar, distribuir, consumir o politizar la energía. Este concepto se relaciona con los distintos vínculos, compromisos o involucramientos sociales en juego en proyectos de energía de todo tipo. El enfoque hacia las formas de implicación permite un acercamiento a las conexiones entre 
la energía -ya sean grandes proyectos eléctricos o prácticas domésticas-y distintos actores (públicos, consumidores, usuarios, comunidades, individuos, instituciones, objetos). Es, por tanto, un planteamiento especialmente rico para observar cómo sociedad y energía se codeterminan a diferentes escalas (macro, meso, micro; local, nacional, global; individual, institucional, sistémico; pasado, presente, futuro).

Los artículos del monográfico revelan distintas formas de involucramiento en la cuestión energética. Primero, la implicación como percepción social: los modos a través de los cuales públicos nacionales se comprometen en proyectos energéticos, guardan relación en cómo los expertos, los usuarios y la población en general imaginan y construyen ideas de 'ciudadano', 'energía' o 'participación'. El artículo de Espluga et al. sobre las percepciones de los actores involucrados en las estrategias de comunicación de implantación y mantenimiento de la energía nuclear en España constituye una muestra de este proceso de construcción social. Oltra y sus colegas, desde otra perspectiva metodológica, se centran en una pregunta similar: qué factores psicosociales determinan la aceptación de hidrógeno y cómo estas actitudes informan acerca de los modos de involucramiento ciudadano que el desarrollo de esta energía debe establecer. El estudio aplicado en 7 países muestra que el nivel de familiaridad con la tecnología del hidrógeno es en general bajo, especialmente, cuando se compara con otras tecnologías alternativas como la energía solar. Sin embargo, los participantes muestran una actitud positiva hacia las tecnologías para la generación de energía con hidrógeno, lo que puede considerarse como un prerrequisito para lograr una mejor adopción. Por su parte, Vancea, Beker y Kunze muestran cómo el éxito en la construcción de proyectos de energía renovable depende en parte de su naturaleza política y colectiva. En particular, los autores señalan la relevancia de los diferentes modos de compromiso y participación voluntaria bajo una tipología de emprendimiento social.

Una segunda línea de reflexión que presenta en el monográfico es la implicación como un modo de gobernanza experimental. Desde esta perspectiva la energía no solo aparece como un espacio relacional donde se crean y consolidan vínculos sociales, sino también como uno donde estos se prueban, testean y especulan a la intemperie. Los proyectos energéticos, sobre todo los vinculados a fuentes renovables no-convencionales, se suelen desarrollar de manera exploratoria y con guías que se articulan sobre la marcha misma de la prospección. Por consiguiente, tales proyectos de innovación tecnológica son espacios ideales para incubar nuevas formas de organización política e identitaria. El artículo de Gross y Tironi, por ejemplo, muestra cómo la inevitable ignorancia sociotécnica que marca la prospección geotermal se traduce en una forma de desarrollo ex- perimental "al descubierto". Este modo experimental modifica la relación de la innovación tecnológica tanto con la lógica científica del laboratorio cómo con las formas del involucramiento ciudadano en la toma de decisiones. En esta misma línea, el artículo de Tironi y Sannazzaro analiza cómo un parque eólico, dada su inherente incertidumbre tecnolegal, abre un espacio para problematizar -o experimentalizar como dicen los autores- una serie de relaciones e implicancias sociales que habían sido, hasta ese momento, asumidas como obvias: el significado de la identidad "indígena" en Chile.

Por último, la tercera línea de reflexión gira en torno a la implicación como relación doméstica, articulada en la relación cotidiana entre artefactos energéticos, usuarios y el diseño de políticas y regulaciones a nivel institucional. En esta clave, Boso, Ariztía y Fonseca analizan el caso de la sustitución de estufas de leña en Temuco, una ciudad intermedia del sur de Chile, con problemas de contaminación atmosférica por la quema de combustible. Los resultados de estudios muestran que en los procesos de aceptación social de tecnologías energéticas emergentes son determinantes tanto los aspectos vinculados con los usos y prácticas que estructuran la vida cotidiana, como los mecanismos de bloqueo que operan a escala institucional.

\section{BREVES CONCLUSIONES}

En resumen, tal como ejemplifican los artículos de este monográfico, la energía se ha vuelto hoy un ámbito de creciente interés y relevancia para las ciencias sociales y, en particular, para la sociología. Esto al menos conlleva dos desafíos. Por una parte, la visibilidad de procesos y transformaciones vinculadas a la energía -tales como el cambio climático, la contaminación atmosférica urbana o los movimientos y fuerzas sociales que dirigen las transiciones energéticas- son acontecimientos y tendencias globales que exigen ser estudiados desde las ciencias sociales. La sociología no puede renunciar a abordar estas temáticas.

Por otra parte, este abordaje plantea el desafío de trascender a distinciones tradicionales en la construcción de objetos sociológicos. Como muestra este monográfico, la energía es un objeto de naturaleza esencialmente sociotécnica y en el que se articulan formas variadas de implicación entre energía y mundo social. En este sentido, los artículos que se presentan ensayan distintas alternativas para afrontar el desafío de pensar la energía más allá de una mirada puramente "ingenieril", proponiendo abordajes que vinculan activamente los distintos elementos técnicos, materiales y sociales que constituyen los fenómenos energéticos. El análisis de este tipo de objetos híbridos deberá consolidarse en los próximos años como un objetivo básico de la agenda sociológica. 


\section{AgradeCIMIEntos}

Los autores agradecen el apoyo de los editores y el equipo de trabajo de la Revista Internacional de Sociología en la elaboración de este monográfico. Este artículo fue financiado por el proyecto Fondecyt
№11150262 y por el Núcleo Milenio de Investigación en Energía y Sociedad. Los autores declaran haber participado por igual en la redacción del artículo, por lo que el orden en el que aparecen es alfabético.

\section{Referencias Bibliográficas}

Alvial-Palavicino, C. y S. Ureta. 2017. "Economizing justice: Turning equity claims into lower energy tariffs in Chile". Energy Policy 105: 642-647. http://doi.org/10.1016/j. enpol.2017.02.013

Ariztía, T. 2017. "La teoría de las prácticas sociales: particularidades, posibilidades y límites". Cinta de Moebio 59: 221-234. http://doi.org/10.4067/S0717554X2017000200221.

Barry, A. 2001. Political Machines: Governing a technological Society. London: Athlone.

Barry, A. 2015. Material Politics: Disputes Along the Pipeline. London: Wiley-Blackwell.

Berti, P. y L. Levidow. 2014. "Fuelling expectations. A policypromise lock-in of UK biofuel policy". Energy Policy 66, 135-143. http://doi.org/10.1016/j.enpol.2013.09.044

Blok, A. 2011. "Clash of the eco-sciences. Carbon marketization, environmental NGOs and performativity as politics". Economy and Society 40(3): 451-476. http://doi. org/10.1080/03085147.2011.574422.

Callon, M. 1980. "The State and Technical Innovation: A Case Study of the Electric Vehicle in France". Research Policy 9: 358-376. http://doi.org/10.1016/00487333(80)90032-3

Cointe, B. 2015. "From a promise to a problem. The political economy of solar photovoltaics in France". Energy Research \& Social Science 8: 151-161. http://doi. org/10.1016/j.erss.2015.05.009

Devine-Wright, P. 2015. Renewable Energy and the Public: From NIMBY to Participation. London and New York: Routledge.

Durkheim, E. 2001 [1895]. Las reglas del método sociológico. México DF: Fondo de Cultura Económica.

Edwards, P. 2003. "Infrastructures and modernity: Forces, time and social organization in the history of sociotechnical systems". Pp. 185-226 en Modernity and Technology, editado por T. Misa, P. Brey y A. Feenberg. Cambridge, MA: MIT Press.

Geels, F. W. 2011. "The multi-level perspective on sustainability transitions. Responses to seven criticisms". Environmental Innovation and Societal Transitions 1(1): 24-40. http://doi.org/10.1016/j.eist.2011.02.002

Geels, F. W. 2014. "Regime Resistance against Low-Carbon Transitions. Introducing Politics and Power into the MultiLevel Perspective". Theory, Culture \& Society 31(5): 21-40. http://doi.org/10.1177/0263276414531627

Geels, F. W., F. Kern, G. Fuchs, N. Hinderer, G. Kungl, J. Mylan et al. 2016. "The enactment of socio-technical transition pathways. A reformulated typology and a comparative multi-level analysis of the German and UK low-carbon electricity transitions (1990-2014)". Research Policy 45(4): 896-913. http://doi.org/10.1016/j. respol.2016.01.015
Guy, S. y E. Shove. 2000. The sociology of energy, buildings and the environment: Constructing knowledge, designing practice, Vol. 5. New York: Psychology Press.

Hecht, G. 2009. The Radiance of France: Nuclear Power and National Identity after World War II. Cambridge, MA: MIT Press.

Howe, C. y D. Boyer. 2015. "Aeolian politics". Distinktion: Scandinavian Journal of Social Theory 16(1): 31-48. http://doi.org/10.1080/1600910X.2015.1022564

Hughes, T. P. 1983. Networks of power. Electrification in Western society, 1880-1930. Softshell books edition. Baltimore: Johns Hopkins University Press.

Jensen, C. B. y B. R. Winthereik. 2013. Monitoring Movements in Development Aid: Recursive Partnerships and Infrastructures. Cambridge, MA: MIT Press.

Larkin, B. 2013. "The Politics and Poetics of Infrastructure". Annual Review of Anthropology 42: 327-343. http://doi. org/10.1146/annurev-anthro-092412-155522

Marres, N. 2012. Material Participation: Technology, the Environment and Everyday Publics. London: Palgrave Macmillan.

McAdam, D. y H. Boudet. 2012. Putting Social Movements in their Place: Explaining Opposition to Energy Projects in the United States, 2000-2005. Cambridge: Cambridge University Press.

MacKenzie, D. 2009. "Making things the same. Gases, emission rights and the politics of carbon markets". Accounting, Organizations and Society 34(3-4): 440-455. http://doi.org/10.1016/j.aos.2008.02.004

McMeekin, A. y D. Southerton. 2012. "Sustainability transitions and final consumption. Practices and socio-technical systems". Technology Analysis \& Strategic Management 24(4): 345-361. http://doi.org/10.1080/09537325 .2012 .663960

Mitchell, T. 2011. Carbon Democracy. Political Power in the Age of Oil. London: Verso.

Pallesen, T. 2016. "Valuation struggles over pricing? Determining the worth of wind power". Journal of Cultural Economy 9(6): 527-540. http://doi.org/10.1080/17530 350.2016.1212084

Rule, T. 2014. Solar, Wind and Land: Conflicts in Renewable Energy Development. London and New York: Routledge.

Shove, E. 2010a. "Social Theory and Climate Change. Questions Often, Sometimes and Not Yet Asked". Theory, Culture \& Society 27(2-3): 277-288. http://doi. org/10.1177/0263276410361498

Shove, E. 2010b. "Beyond the ABC. Climate change policy and theories of social change". Environ. Plann. A 42(6): 1273-1285. http://doi.org/10.1068/a42282

Shove, E.y G. Walker. 2014. "What Is Energy For? Social Practice and Energy Demand". Theory, Culture \& Society 31(5): 41-58. http://doi.org/10.1177/0263276414536746 
Spaargaren, G. 2011. "Theories of practices. Agency, technology, and culture". Global Environmental Change 21(3): 813-822. http://doi.org/10.1016/j.gloenvcha.2011.03.010

Star, S. L. 1999. "The ethnography of infrastructure". American Behavioral Scientist 43(3): 377-391. http://doi. org/10.1177/00027649921955326

Star, S. L. y K. Ruhleder. 1996. "Steps toward an ecology of infrastructure: Design and access for large information spaces". Information Systems Research 7(1): 111-134. http://doi.org/10.1287/isre.7.1.111

Stern, P. C. 2014. "Individual and household interactions with energy systems: toward integrated understanding". Energy Research \& Social Science 1: 41-48. http://doi. org/10.1016/j.erss.2014.03.003

Summerton, J. 2004. "Do Electrons Have Politics? Constructing User Identities in Swedish Electricity". Science, Technology \& Human Values 29(4): 486-511. http://doi. org/10.1177/0162243904264487
Tironi, M. y J. Barandiaran. 2014. "Neoliberalism as political technology: expertise, energy and democracy in Chile". Pp 303-327 en Beyond Imported Magic: Studying Science and Technology in Latin America, editado por E. Medina, I. Costa Márques y C. Holmes. Cambridge, MA: MIT Press.

Upham, P., C. Oltra, À., Boso 2015. "Towards a cross-paradigmatic framework of the social acceptance of energy systems". Energy Research \& Social Science 8: 100112 . http://doi.org/10.1016/j.erss.2015.05.003

Varas, P., M. Tironi, H. Rudnick y N. Rodríguez. 2013. "The growing social challenges of hydroelectric development". IEEE Power \& Energy 11(3): 66-75. http://doi. org/10.1109/MPE.2013.2245586

Wilkie, A., M. Michael y M. Plummer-Fernandez. 2015. "Speculative Method and Twitter: Bots, Energy and Three Conceptual Characters". Sociological Review 63(1): 79-101. http://doi.org/10.1111/1467-954X.12168

Zélem, M. C. y C. Beslay. 2015. Sociologie de l'énergie: gouvernance et pratiques sociales. Paris: CNRS éditions.

TOMÁS ARIZTíA es Sociólogo. PhD en Sociología, London School of Economics and Political Science. Profesor Asociado de la Escuela de Sociología Universidad Diego Portales y Director del Magister en Métodos para la Investigación Social de esta misma casa de Estudios. Investigador Responsable Suplente del Núcleo Milenio en Energía y Sociedad (NUMIES). Sus intereses de investigación abarcan el entrecruce de tres áreas: la sociología del consumo sustentable, la sociología económica y la sociología del conocimiento. Ha publicado sobre estos temas en diversas revistas internacionales de sociología como el Sociological Review, Enviroment and Planning A, Journal of Consumer Culture y Journal of Cleaner Production. Actualmente coordina el área de "usos de energía" del Núcleo Milenio en Energía y Sociedad en la cual está realizando investigación etnográfica sobre las prácticas e infraestructuras de uso energético en los hogares y las dinámicas de transformación y comercialización de nuevas infraestructuras domesticas para el uso de energía.

ÀLEX BOSO es Doctor por el Departamento de Teoría Sociológica, Filosofía del Derecho y Metodología de las Ciencias Sociales de la Universidad de Barcelona. Es profesor e investigador del Núcleo Científico Tecnológico en Ciencias Sociales y Humanidades de la Universidad de la Frontera, Temuco, Chile. Ha sido profesor asociado del Departamento de Ciencias Políticas y Sociales de la Universidad Pomeu Fabra y de la Facultad de Salud y Acción Social de la Universidad de Vic. Ha colaborado como investigador en el Centro de Investigación Sociotécnica del CIEMAT y en Internet Interdisciplinary Institute de la Universitat Oberta de Catalunya. Sus principales áreas de estudio son el análisis de las políticas públicas y las relaciones entre salud, medioambiente y migraciones. Sus trabajos han sido publicados en diversas revistas de relevancia académica, entre las que cabe destacar: Energy Policy; Energy and Environtment; Greenhouse Gases: Science and Technology; Energy Research \& Social Sicence; Basic Income Studies o Critical Social Policy. Actualmente dirige una investigación sobre procesos de implicación ciudadana en el control de la contaminación atmosférica de ciudades intermedias del sur de Chile.

MANUEL TIRONI es profesor asociado y coordinador del grupo Estudios Críticos del Antropoceno en el Instituto de Sociología de Universidad Católica de Chile. Es investigador asociado del Centro de Investigación Integrada en Desastres Naturales (CIGIDEN) y del Núcleo Milenio de Investigación en Energía y Sociedad (NUMIES). Su trabajo se ubica en la intersección entre los estudios sociales de la ciencia y tecnología, la sociología medioambiental y la teoría política. Sus últimos proyectos tratan temas de toxicidad, justicia ambiental, conocimiento geológico y ciencia ciudadana. Sus artículos más recientes han sido publicados en Sociological Review, Geoforum y Science Technology \& Human Values, entre otros. Ha sido profesor invitado en las universidades de Goldsmiths - University of London, Copenhague y Warwick. 